\title{
A Phase 4, Open-Label, Single-Arm Study Assessing the Efficacy and Safety of Ivabradine in African American Patients with Heart Failure and Reduced Ejection Fraction
}

David E. Lanfear - Kelsey R. Neaton · Chen Lu • Yimeng Liu •

Ricardo E. Dent-Acosta

Received: July 15, 2020 / Published online: August 17, 2020

(C) The Author(s) 2020

\section{ABSTRACT}

Introduction: There are limited data on ivabradine therapy in black patients and none in African Americans. We performed an open-label, prospective study at two centers in the United States. African American patients with heart failure (HF) $(N=30)$, left ventricular ejection fraction $\leq 35 \%$, and in sinus rhythm with resting heart rate $(\mathrm{HR}) \geq 70 \mathrm{bpm}$ received ivabradine $2.5-7.5 \mathrm{mg}$ twice daily for 57 days.

Digital Features To view digital features for this article go to https://doi.org/10.6084/m9.figshare.12746042.

Electronic supplementary material The online version of this article (https://doi.org/10.1007/s40119020-00196-1) contains supplementary material, which is available to authorized users.

D. E. Lanfear $(\varangle) \cdot$ K. R. Neaton

Henry Ford Health System, Detroit, MI, USA

e-mail: DLANFEA1@hfhs.org

K. R. Neaton

e-mail: KNeaton1@hfhs.org

C. Lu · Y. Liu · R. E. Dent-Acosta

Amgen Inc., Thousand Oaks, CA, USA

C. $\mathrm{Lu}$

e-mail: chelu@amgen.com

Y. Liu

e-mail: yliu03@amgen.com

R. E. Dent-Acosta

e-mail: rdent@amgen.com
Methods: The primary endpoint was change in HR from baseline to day 57, compared with the $-5 \mathrm{bpm}$ change observed in the absence of ivabradine in the placebo group of the SHIFT study. The safety endpoint was treatmentemergent adverse events (TEAEs). Exploratory endpoints were change from baseline to day 57 in 6-minute walk test (6MWT) distance, HR difference during a 6MWT (i.e. HR at minute 6 - resting HR), and physical activity counts.

Results: At day 57, the estimated least squares mean change from baseline in $\mathrm{HR}$ was $-9.5 \mathrm{bpm}(95 \% \mathrm{CI}-13.0,-6.0)$. The estimated mean treatment difference with ivabradine versus a presumed $-5 \mathrm{bpm}$ change from baseline $H R$, as seen in the placebo group of the SHIFT study, was $-4.5 \mathrm{bpm}(95 \% \mathrm{CI}-8.0,-1.0$; $p=0.013$ ). The mean (SE) changes in $6 \mathrm{MWT}$ distance and HR difference during the 6MWT were 16.3 (10.8) meters and 2.3 (3.7) bpm, respectively. Ivabradine therapy did not result in greater physical activity. TEAEs were reported in $11(36.7 \%)$ patients.

Conclusion: These data support ivabradine use in African American patients with HF with reduced ejection fraction who meet typical treatment criteria.

Trial Registration: ClinicalTrials.gov identifier, NCT03456856

Keywords: Efficacy; Heart failure; Ivabradine; Safety 


\section{Key Summary Points}

Why carry out this study?

There are limited data on ivabradine therapy in black patients and none in African Americans.

The aim of the current study was to assess the effect of ivabradine on heart rate (HR) and physical activity when added to guideline-directed therapy in African American patients with heart failure with reduced ejection fraction (HFrEF).

\section{What was learned from the study?}

Ivabradine $2.5-7.5 \mathrm{mg}$ twice daily demonstrated effective HR reduction from baseline in African American patients with HFrEF.

These data support ivabradine use in African American patients with HFrEF who meet typical treatment criteria.

\section{INTRODUCTION}

Chronic heart failure (HF) is a complex syndrome that results in inadequate systemic blood flow when neurohormonal mechanisms (i.e. the sympathetic nervous system and the renin-angiotensin-aldosterone system) are no longer able to deliver an adequate physiological response [1]. African American patients experience a $50 \%$ higher rate of HF relative to the general population and have an increased risk of mortality secondary to chronic HF compared with white patients [2, 3]. Further, elevated heart rate (HR) is associated with increased HFrelated hospitalizations and mortality in African Americans [4]. Beta-blockers may be of limited benefit to African American patients with HF with reduced ejection fraction (HFrEF) $[5,6]$. Thus, the development and evaluation of alternative mechanisms of ventricular rate reduction is warranted in this population.
Ivabradine is a hyperpolarization-activated cyclic nucleotide-gated channel blocker that reduces the spontaneous pacemaker activity of the cardiac sinus node by selectively inhibiting the cardiac f-current $\left(I_{\mathrm{f}}\right)$, resulting in HR reduction with no effects on ventricular repolarization and myocardial contractility [7-9]. Ivabradine is approved in the United States (US) to reduce the risk of hospitalization for worsening HF in adult patients with stable, symptomatic HFrEF, and for the treatment of stable symptomatic HF due to dilated cardiomyopathy in pediatric patients aged 6 months and older [7]. The American College of Cardiology (ACC)/American Heart Association (AHA) clinical guidelines recommend that ivabradine be used in adult patients with symptomatic (New York Heart Association [NYHA] Class II-III) stable chronic HFrEF who are receiving guideline-directed management and therapy, including a beta-blocker at maximum tolerated dose, and who are in sinus rhythm with an HR of $\geq 70$ beats per minute (bpm) at rest [2].

Despite being disproportionately affected by HF $[2,3]$, African American patients have been underrepresented in cardiovascular drug trials to date [10], including ivabradine studies that informed both its current US indication and inclusion in the ACC/AHA guidelines $[7-9,11,12]$. For instance, $98.1 \%$ and $89 \%$ of patients included in the BEAUTIFUL and SHIFT studies of ivabradine, respectively, were white, and both studies were conducted outside the US $[8,9]$. Consequently, the efficacy and safety of ivabradine in African American patients with HFrEF remains to be elucidated. Therefore, the aim of the current study was to assess the effect of ivabradine on HR and physical activity when added to guideline-directed therapy in African American patients with HFrEF.

\section{METHODS}

\section{Study Design and Patients}

This was an open-label, prospective, single-arm phase 4 study conducted at two centers in the US (ClinicalTrials.gov identifier: NCT0345 6856). Eligible patients were aged $\geq 18$ years, self-described as African American or black, had 
a diagnosis of HF confirmed by medical records, and were in a stable condition and being treated with stable, optimal pharmacological therapy; both study centers were requested to optimize standard of care therapy per Good Clinical Practice. Additionally, eligible patients had a left ventricular ejection fraction (LVEF) $\leq 35 \%$, NYHA Class II-IV HF at screening, and electrocardiogram documentation at the time of screening of sinus rhythm with resting $\mathrm{HR} \geq 70 \mathrm{bpm}$. The main exclusion criteria included a recent myocardial infarction ( $\leq 2$ months) or stroke ( $\leq 1$ month) prior to enrollment.

After a 7-day screening period, eligible patients entered a 57-day open-label treatment period with a starting dose of ivabradine $5 \mathrm{mg}$ twice daily (BID) (Supplementary Fig. S1). Patients with a history of conduction defects, or in whom bradycardia could lead to hemodynamic compromise, received ivabradine $2.5 \mathrm{mg}$ BID. On day 15 , dose titration was conducted according to HR per the US Food and Drug Administration prescribing information for ivabradine [7], up to a maximum dose of $7.5 \mathrm{mg}$ BID (Supplementary Table S1). Ivabradine doses could be adapted to HR at any clinical visit, if necessary. An accelerometer was used to record continuous daytime physical activity from baseline until the end of treatment. This device recorded continuous parameters of physical activity, with additional physical activity data obtained from a standard 6-minute walk test (6MWT) performed at baseline and at the end of treatment; an HR monitor was used to assess HR response during the 6MWT. Following treatment, safety follow-up was conducted at day 87 or 30 days after the last treatment (Supplementary Fig. S1).

\section{Endpoints}

The primary efficacy endpoint was the change in HR from baseline to day 57. This was compared with the $-5 \mathrm{bpm}$ change observed in the overall placebo group of the SHIFT study [9]. The primary efficacy endpoint was also assessed in the following patient subgroups: baseline $\mathrm{HR} \geq 77 \mathrm{bpm} /<77 \mathrm{bpm} ; \quad$ age $\geq 65$ years/
$<65$ years; women/men. The safety endpoint was treatment-emergent adverse events (TEAEs) coded using version 21.1 of the Medical Dictionary for Regulatory Activities (MedDRA); severity assessments were made using the National Cancer Institute Common Terminology Criteria for Adverse Events (CTCAE), version 4.03. Exploratory endpoints included change in 6MWT distance (6MWD) from baseline to day 57 , change in HR difference during a 6MWT (i.e. HR at minute 6 of the $6 \mathrm{MWT}-$ resting HR) from baseline to day 57 , and change in physical activity counts from baseline to day 57 measured by an accelerometer.

\section{Statistical Analysis}

All statistical analyses were conducted with SAS version 9.4 software (SAS, Cary, NC, USA). Continuous variables were summarized using descriptive statistics, including the number of observations $(n)$, mean, standard deviation (SD) or standard error (SE), median, the first and third quartiles, minimum, and maximum. Categorical variables were summarized using the number and percent of patients. Efficacy analyses were performed on the full analysis set, which included all enrolled patients. Summary statistics and least squares (LS) means along with 95\% confidence intervals (CIs), analyzed by a repeated-measures linear model, were estimated for the primary endpoint. The model included terms of scheduled visits and baseline HR measurement as covariates and tested whether the mean reduction with ivabradine exceeded $5 \mathrm{bpm}$, which was the reduction assumed with placebo. Missing values were not imputed. Efficacy analyses in patient subgroups (baseline $\mathrm{HR} \geq 77 \mathrm{bpm} /<77 \mathrm{bpm} ;$ age $\geq 65$ years/ $<65$ years; women $/$ men) used the same model as the primary endpoint. A sensitivity analysis of the primary endpoint was performed using an analysis of covariance model, which included age, sex, and baseline HR as covariates. Safety analyses were performed on the safety analysis set, which included all enrolled patients who received at least one dose of ivabradine. Subject incidence of TEAEs was tabulated by system organ class and preferred 
term. Analyses of safety laboratory endpoints included summary statistics at baseline.

\section{Compliance with Ethics Guidelines}

This study was conducted in accordance with the Declaration of Helsinki, International Council for Harmonisation (ICH), Good Clinical Practice (GCP) and Food and Drug Administration (FDA) regulations/guidelines. The institutional review boards (IRBs) and independent ethics committees (IECs) for this study reviewed the study protocol, amendments, and the informed consent form (ICF). The IRBs that approved the study were the Henry Ford Health System IRB (Detroit, MI, USA) and the Advarra IRB (Columbia, MD, USA). No subjects were recruited into the study and no investigational product was shipped until the IRB/IEC gave written approval of the protocol and ICF and Amgen received copies of these approvals. Informed consent was obtained from all individual participants included in the study.

\section{RESULTS}

All enrolled patients [ $N=30(100 \%)]$ received at least one dose of ivabradine, and 27 (90\%) patients completed the study. The mean (SD) age of the patients was 55.7 (12.6) years, 70\% were male, and the mean (SD) LVEF was $25.6 \%$ (6.8). The majority of patients (73\%) had NYHA Class II HF (Table 1). A beta-blocker was used by $27(90 \%)$ patients, and $18(60 \%)$ patients were receiving an angiotensin-converting enzyme inhibitor. Fifteen $(50 \%)$ patients were taking diabetes medications (Supplementary Table S2).

Three (10\%) patients did not have ivabradine dose changes, 24 (80\%) patients had one dose change, and $3(10 \%)$ patients had two or three dose changes. Twenty-seven (90\%) patients received up-titration from ivabradine $5 \mathrm{mg}$ BID to ivabradine $7.5 \mathrm{mg} \mathrm{BID}$, and 3 (10\%) patients received down-titration from ivabradine $7.5 \mathrm{mg}$ BID to $5 \mathrm{mg}$ BID. The overall mean (SD) exposure to ivabradine was $2.0(0.4)$ months; all patients were exposed to ivabradine for $\geq 2$ weeks, $\quad 29 \quad(96.7 \%) \quad$ patients
Table 1 Baseline demographics and clinical characteristics

\begin{tabular}{ll}
\hline Characteristic & $\begin{array}{l}\text { Ivabradine } \\
(N=30)\end{array}$ \\
\hline Sex, $n$ (\%) & $21(70.0)$ \\
Male & $9(30.0)$ \\
Female & \\
Age (years) & $55.7(12.6)$ \\
Mean (SD) & $24(80.0)$ \\
18-64 years, $n$ (\%) & $6(20.0)$ \\
$\geq$ 65 years, $n$ (\%) & $126.8(17.1)$ \\
Systolic blood pressure (mmHg), & \\
mean (SD) & $73.0(5.7)$ \\
Diastolic blood pressure (mmHg), & \\
mean (SD) & \\
Heart rate (beats per minute), mean & $83.6(8.9)$ \\
(SD) & \\
B-type natriuretic peptide, median & $136(48,325)$ \\
(Q1, Q3) & \\
NYHA class, $n$ (\%) & \\
Class II & \\
Class III & \\
\hline
\end{tabular}

LVEF left ventricular ejection fraction, NYHA New York Heart Association, $Q$ quartile, $S D$ standard deviation

for $\geq 4$ weeks, and $25 \quad(83.3 \%)$ patients for $\geq 8$ weeks.

\section{Change in HR with Ivabradine}

The mean (SD) baseline HR was 83.6 (8.9) bpm (Table 1). Within 2 weeks, HR was lowered by $10 \mathrm{bpm}$ and this was sustained to day 57 (Fig. 1); the mean (SD) HR at day 57 was 73.5 (11.5) bpm. At day 57, the estimated LS mean change from baseline in HR was $-9.5 \mathrm{bpm}$ (95\% CI 


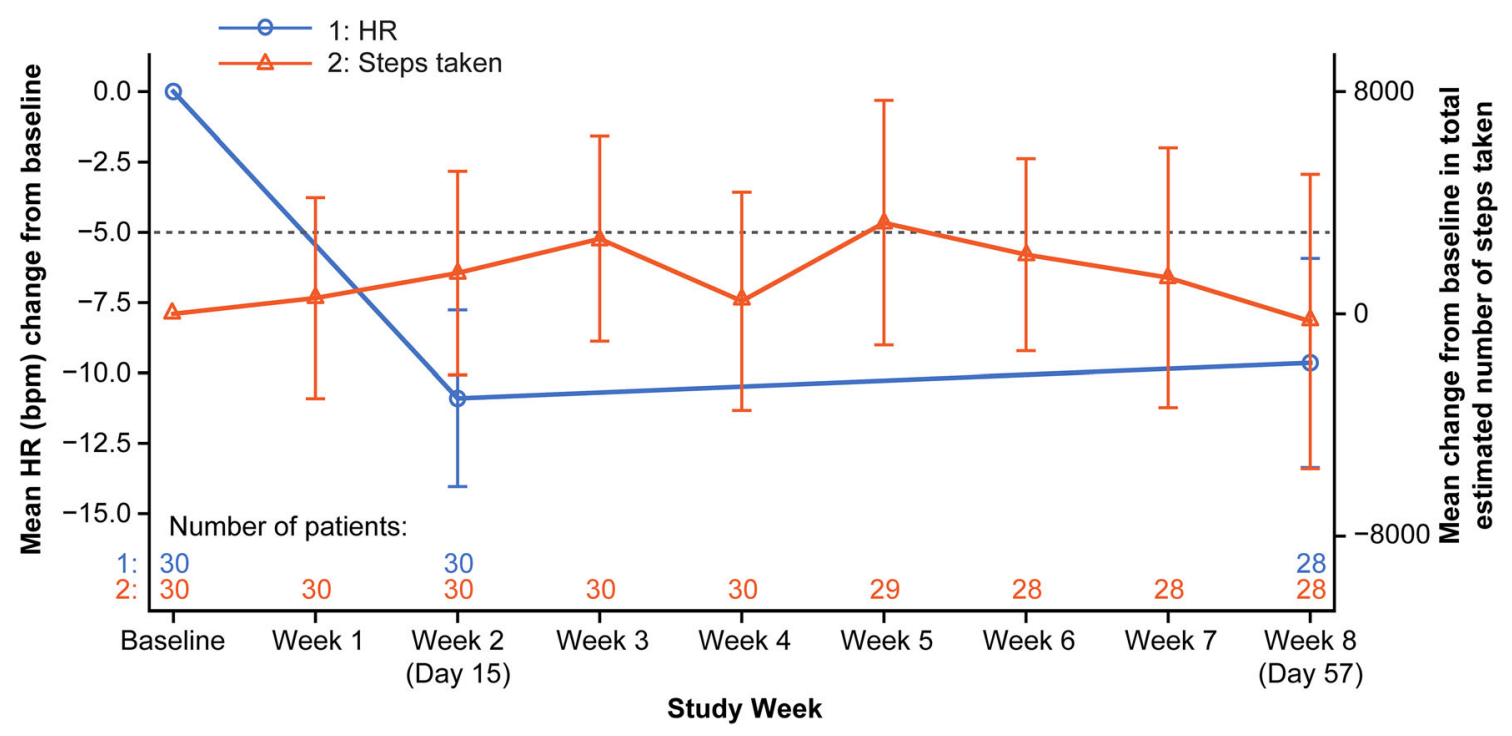

Fig. 1 Mean change in heart rate and total estimated number of steps taken from baseline to end of treatment. Reference line is plotted at $-5 \mathrm{bpm}$ as the change in HR from baseline observed in the placebo group in the SHIFT study. Vertical lines represent the $95 \%$ confidence intervals

$-13.0,-6.0)$. The estimated mean treatment difference with ivabradine versus a presumed $-5 \mathrm{bpm}$ change from baseline HR was $-4.5 \mathrm{bpm}$ (95\% CI $-8.0,-1.0 ; p=0.013)$. The results of the sensitivity analysis were consistent with the primary analysis: estimated mean treatment difference $-4.6 \mathrm{bpm} \quad(95 \%$ CI $-7.7,-1.6$; $p=0.005)$. Similar results were observed between the subgroups of patients with baseline $\mathrm{HR}<77 \mathrm{bpm}$ versus $\geq 77 \mathrm{bpm}$, while the estimated LS mean change from baseline in HR was greater in patients aged $\geq 65$ years versus $<65$ years, and in women versus men (Supplementary Table S3).

\section{Changes in 6MWD, HR Difference During the 6MWT, and Physical Activity}

At baseline, the mean (SE) 6MWD was 172.4 (13.3) meters. At day 57, the mean (SE) change from baseline in 6MWD was 16.3 (10.8) meters (Supplementary Fig. S2), which was not statistically significant $(p=0.141)$. At baseline, the mean (SE) HR difference during the 6MWT was 12.7 (2.3) bpm, and the mean (SE) change from around the means. Missing values were not imputed. Total estimated number of steps taken is defined as the sum of daily total estimated steps taken in 7 days prior to/on the target study day. $b p m$ beats per minute, $H R$ heart rate

baseline to day 57 in HR difference during the 6MWT was 2.3 (3.7) bpm (Supplementary Fig. S2). At day 57, there were no significant changes from baseline in total estimated steps taken (Fig. 1) or proportion of time spent in different physical activity categories (Supplementary Fig. S3).

\section{Safety}

Overall, TEAEs were reported in 11 (36.7\%) patients, and most were CTCAE grade 1 or 2 in severity. The most common TEAEs were dizziness [4 (13.3\%) patients], headache [2 (6.7\%) patients], and cough [2 (6.7\%) patients]. No visual disturbances were reported. One (3.3\%) patient reported a nonserious TEAE of fatigue leading to withdrawal of ivabradine. There were no fatal events (Supplementary Table S4).

\section{DISCUSSION}

In the current study, we observed that ivabradine $2.5-7.5 \mathrm{mg}$ BID treatment effectively 
reduced HR from baseline in African American patients with HFrEF, with a significant treatment effect compared with the $-5 \mathrm{bpm}$ change observed in the placebo group of the SHIFT study [9]. It is important to note that baseline HR was higher in the current study than in the SHIFT study $(83.6 \mathrm{bpm}$ vs. $79.9 \mathrm{bpm}$, respectively) [9], which may explain the larger treatment effect seen here. Previously, a small but significant improvement in NYHA class was observed with ivabradine treatment in the SHIFT study [9]. Here, we observed an improvement in 6MWD with ivabradine therapy, but there was no sustained change in physical activity as measured by an accelerometer. This could suggest that the reduction in HR from baseline did not directly confer an increase in physical activity during daily life, but these were exploratory endpoints and the study may not have been adequately powered to rule out an effect. Additional studies would be needed to assess the effect of ivabradine on physical activity outcomes in African American patients with HFrEF. Reassuringly, we witnessed no new safety signals in this study compared with the established safety profile of ivabradine $[7,13]$.

ACC/AHA clinical guidelines for ivabradine are based on studies in which patients with African ancestry were underrepresented or absent $[2,8,9,11,12]$, and the paucity of data to support treatment guidelines for African Americans with HFrEF was recognized in an ACC expert consensus statement as a pivotal issue [14]. This insufficient knowledge base regarding medication effect is even more challenging given the higher prevalence of certain comorbid conditions (e.g. chronic kidney disease, diabetes, amyloidosis), and more limited access to care, in African American patients with HFrEF [15]. As the first study to report the efficacy and safety of ivabradine specifically in self-identified African American patients with HFrEF, the current work represents a valuable addition to the literature and has the potential to inform clinical guidelines [2]. While hard clinical outcomes were not assessed, the efficacy in terms of HR lowering (which is thought to be the sole mechanism of action of ivabradine) suggests that current ACC/AHA guideline recommendations for ivabradine treatment could be generalized to African American patients with HFrEF [2]. However, while the current results are encouraging, future studies that include additional cardiovascular endpoints are needed to further support the efficacy and safety of ivabradine in the African American population.

Limitations of the current study include the lack of a placebo arm and the use of placebo data from the SHIFT trial [9]. While this is understandable based on the population, and the post-approval status of ivabradine, it resulted in some baseline characteristics not being well-matched. For example, compared with the SHIFT trial, the baseline HR in the current study was slightly higher and patients in the current study were younger [9]. Additionally, regulatory endpoints such as mortality and hospitalization due to HF were not included. Lastly, the sample size was relatively small, and larger studies are warranted in this population.

\section{CONCLUSION}

In conclusion, we conducted this open-label, single-arm interventional study, as African American patients were not enrolled in previous ivabradine clinical trials. Ivabradine $2.5-7.5 \mathrm{mg}$ BID demonstrated effective HR reduction from baseline in African American patients with HFrEF. No new patterns indicative of clinically important TEAEs were observed, and no new safety risks were identified.

\section{ACKNOWLEDGEMENTS}

The authors would like to thank the participants of the study.

Funding. The study and the journal's Rapid Service Fee were funded by Amgen Inc. (Thousand Oaks, CA, USA).

Authorship. All named authors meet the International Committee of Medical Journal Editors (ICMJE) criteria for authorship for this article, take responsibility for the integrity of the work as a whole, and have given their 
approval for this version to be published. This manuscript is dedicated to the memory of the principal investigator at the second study site, Dr. Alan Meholick (Buffalo, NY, USA), who died prior to development of this manuscript.

Medical Writing, Editorial, and Other Assistance. Medical writing support, in accordance with Good Publication Practice (GPP3) guidelines, was provided by Liam Gillies, PhD, of Cactus Life Sciences (part of Cactus Communications), and funded by Amgen Inc.

Disclosures. David E. Lanfear reports research funding from Amgen, Bayer, the NHLBI, Novartis, and Critical Diagnostics, and is a consultant to Amgen, Abbot Diagnostics, Abiomed, Ortho Diagnostics, Janssen, Hridaya, and the Duke Clinical Research Institute (Akros, Novartis). Kelsey R. Neaton has nothing to disclose. Chen Lu, Yimeng Liu, and Ricardo E. Dent-Acosta are employees of and shareholders in Amgen Inc.

Compliance with Ethics Guidelines. This study was conducted in accordance with the Declaration of Helsinki, International Council for Harmonisation (ICH), Good Clinical Practice (GCP), and Food and Drug Administration (FDA) regulations/guidelines. The institutional review boards (IRBs) and independent ethics committees (IECs) for this study reviewed the study protocol, amendments, and the informed consent form (ICF). The IRBs that approved the study were the Henry Ford Health System IRB (Detroit, MI, USA) and the Advarra IRB (Columbia, MD, USA). No subjects were recruited into the study and no investigational product was shipped until the IRB/IEC gave written approval of the protocol and ICF and Amgen received copies of these approvals. Informed consent was obtained from all individual participants included in the study.

Data Availability. Qualified researchers may request data from Amgen clinical studies. Complete details are available at the following: https://www.amgen.com/datasharing.
Open Access. This article is licensed under a Creative Commons Attribution-NonCommercial 4.0 International License, which permits any non-commercial use, sharing, adaptation, distribution and reproduction in any medium or format, as long as you give appropriate credit to the original author(s) and the source, provide a link to the Creative Commons licence, and indicate if changes were made. The images or other third party material in this article are included in the article's Creative Commons licence, unless indicated otherwise in a credit line to the material. If material is not included in the article's Creative Commons licence and your intended use is not permitted by statutory regulation or exceeds the permitted use, you will need to obtain permission directly from the copyright holder. To view a copy of this licence, visit http://creativecommons.org/licenses/by$\mathrm{nc} / 4.0 /$.

\section{REFERENCES}

1. Francis GS. Pathophysiology of chronic heart failure. Am J Med. 2001;110(Suppl 7A):37S-46S.

2. Yancy CW, Jessup M, Bozkurt B, et al. 2017 ACC/ AHA/HFSA focused update of the 2013 ACCF/AHA guideline for the management of heart failure: a report of the American College of Cardiology/ American Heart Association Task Force on Clinical Practice Guidelines and the Heart Failure Society of America. J Am Coll Cardiol. 2017;70:776-803.

3. Sidney S, Quesenberry CP Jr, Jaffe MG, et al. Recent trends in cardiovascular mortality in the United States and public health goals. JAMA Cardiol. 2016;1(5):594-9.

4. Parikh KS, Greiner MA, Suzuki T, et al. Resting heart rate and long-term outcomes among the African American population: insights from the Jackson Heart Study. JAMA Cardiol. 2017;2:172-80.

5. Beta-Blocker Evaluation of Survival Trial Investigators, Eichhorn EJ, Domanski MJ, Krause-Steinrauf $\mathrm{H}$, Bristow MR, Lavori PW. A trial of the betablocker bucindolol in patients with advanced chronic heart failure. N Engl J Med. 2001;344(22): 1659-67.

6. Lanfear DE, Hrobowski TN, Peterson EL, et al. Association of beta blocker exposure with outcomes in heart failure differs between African American 
and white patients. Circ Heart Fail. 2012;5(2): 202-8.

7. Amgen Inc. CORLANOR ${ }^{\circledR}$ (ivabradine) [package insert]. U.S. Food and Drug Administration website. https://www.accessdata.fda.gov/drugsatfda_docs/ label/2019/209964lbl.pdf. Revised Apr 2019. Accessed Aug 2020.

8. Fox K, Ford I, Steg PG, Tendera M, Ferrari R, BEAUTIFUL Investigators. Ivabradine for patients with stable coronary artery disease and left-ventricular systolic dysfunction (BEAUTIFUL): a randomised, double-blind, placebo-controlled trial. Lancet. 2008;372(9641):807-16.

9. Swedberg K, Komajda M, Böhm M, et al. Ivabradine and outcomes in chronic heart failure (SHIFT): a randomised placebo-controlled study. Lancet. 2010;376(9744):875-85.

10. Ortega RF, Yancy CW, Mehran R, Batchelor W. Overcoming lack of diversity in cardiovascular clinical trials: a new challenge and strategies for success. Circulation. 2019;140(21):1690-2.

11. Fox K, Ford I, Steg PG, et al. Ivabradine in stable coronary artery disease without clinical heart failure. N Engl J Med. 2014;371(12):1091-9.
12. Böhm M, Robertson M, Ford I, et al. Influence of cardiovascular and noncardiovascular co-morbidities on outcomes and treatment effect of heart rate reduction with ivabradine in stable heart failure (from the SHIFT Trial). Am J Cardiol. 2015;116(12): 1890-7.

13. Savelieva I, Camm AJ. $I_{\mathrm{f}}$ inhibition with ivabradine: electrophysiological effects and safety. Drug Saf. 2008;31(2):95-107.

14. Yancy CW, Januzzi JL Jr, Allen LA, et al. 2017 ACC Expert Consensus Decision Pathway for Optimization of Heart Failure Treatment: Answers to 10 Pivotal Issues About Heart Failure with Reduced Ejection Fraction: a Report of the American College of Cardiology Task Force on Expert Consensus Decision Pathways. J Am Coll Cardiol. 2018;71(2): 201-30.

15. Tillman F, Kim J, Makhlouf T, Osae L. A comprehensive review of chronic heart failure pharmacotherapy treatment approaches in African Americans. Ther Adv Cardiovasc Dis. 2019;13: 1753944719840192. 